\title{
Entanglement of Square Nets in Covalent Organic Frameworks
}

\author{
Fangying Jin, ${ }^{\dagger, \diamond}$ Ha L. Nguyen, ${ }^{\dagger, \ddagger}{ }^{\ddagger}$ Zhiye Zhong, ${ }^{\nabla}$ Xing Han, ${ }^{\dagger}$ Chenhui Zhu, ${ }^{\varnothing}$ Xiaokun Pei, ${ }^{\dagger}$ Yan- $^{-}$ \\ hang Ma, ${ }^{\nabla}$ and Omar M. Yaghi ${ }^{*},+\neq$ \\ ${ }^{\dagger}$ Department of Chemistry, University of California-Berkeley; Kavli Energy Nanoscience Institute at UC Berkeley; \\ and Berkeley Global Science Institute, Berkeley, California 94720, United States \\ ${ }^{\ddagger}$ Joint UAEU-UC Berkeley Laboratories for Materials Innovations, United Arab Emirates University, Al-Ain 15551, \\ United Arab Emirates \\ ${ }^{\nabla}$ School of Physical Science and Technology, ShanghaiTech University, Shanghai 201210, China \\ ${ }^{\varnothing}$ Advanced Light Source, Lawrence Berkeley National Laboratory, Berkeley, California 94720, United States
}

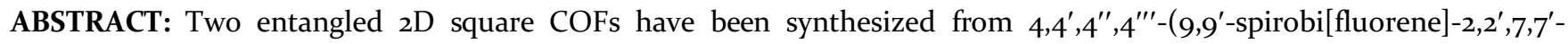
tetrayl)-tetrabenzaldehhyde (SFTB) and $p$-phenylenediamine (PPA) and benzidine (BZD) to form COF-38, $\left[(\mathrm{SFTB})(\mathrm{PPA})_{2}\right]_{\text {imine }}$ and its isoreticular form COF-39, $\left[(\mathrm{SFTB})(\mathrm{BZD})_{2}\right]_{\text {imine }}$. We also report the single crystal electron diffraction structure of COF-39 and find that it is composed of mutually entangled $2 \mathrm{D}$ square nets (sql). These COFs represent the first examples of entangled $2 \mathrm{D}$ COF structures, which as we illustrate were made possible by our strategy of using the distorted tetrahedral SFTB building unit. SFTB overcomes the propencity of $2 \mathrm{D}$ COFs to stack through $\pi-\pi$ stacking and allows for entanglements to form. This work adds significantly to the design principles of COFs.
\end{abstract}

Covalent organic frameworks (COFs) are a class of extended structures formed by linking organic building units through strong covalent bonds. ${ }^{1-9} 2 \mathrm{D}$ COFs are often constructed by the combination of planar linking units facilitated by $\pi-\pi$ stacking. ${ }^{3,5}$ For $3 \mathrm{D}$ COFs, the vast majority uses non-planar a)
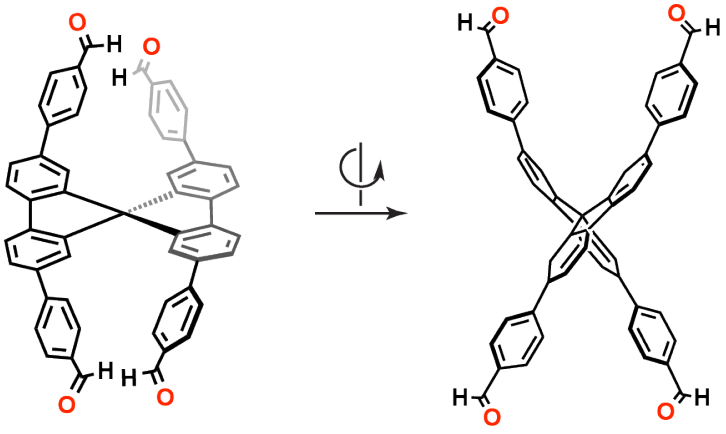

4,4',4",4'"-(9,9'-Spirobi[fluorene]-2,2',7,7'-tetrayl) -tetrabenzaldehyde (SFTB) b)

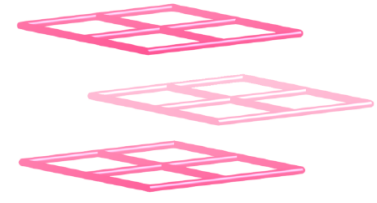

Square nets (AB stacking)

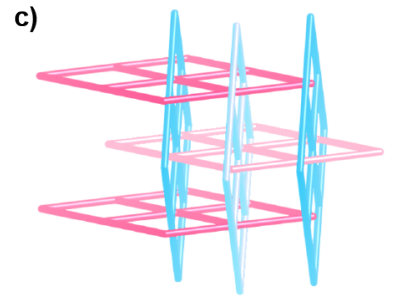

Entanglement of square nets
Figure 1. (a) Chemical structure of $4,4^{\prime}, 4^{\prime \prime}, 4^{\prime \prime \prime}-\left(9,9^{\prime}-\right.$ spirobi[fluorene]-2,2',7,7'-tetrayl)-tetrabenzaldehhyde (SFTB). Illustration of (b) AB stacked square nets and (c) entanglement of square nets. units ${ }^{10-12}$ to produce mainly bor, ${ }^{13}$ ctn, ${ }^{13}$ dia,,${ }^{14}$ pts, ${ }^{15}$ lon, ${ }^{16}$ acs, ${ }^{17}$ bcu, ${ }^{18} \mathbf{~ s t p}^{19} \mathbf{l j h}^{20}$ and ceq $^{21}$ topologies. Although entanglements are well-known for $3 \mathrm{D}$ COFs, ${ }^{15,17,22-24}$ they remain unknown for $2 \mathrm{D}$ COFs. The persistance of this state of affairs is likely due to the fact that the formation of $2 \mathrm{D}$ COFs has relied entirely on choosing planar building blocks and deploying $\pi-\pi$ stacking as means of organizing and ultimately crystallizing the material. ${ }^{25-27}$ In this report, we show how a distorted tetrahedral building block could be used to prevent $\pi-\pi$ stacking and allow for $2 \mathrm{D}$ COF entanglements to form, and therefore increase the dimensionality of COFs from $2 \mathrm{D}$ to 3D.

Our strategy is based on using $\left(4,4^{\prime}, 4^{\prime \prime}, 4^{\prime \prime \prime}-\left(9,9^{\prime}-\right.\right.$ spirobi[fluorene]-2,2',7,7'-tetrayl)-tetrabenzaldehhyde, SFTB, Figure 1a) as a building unit. Although the central carbon atom is tetrahedral, the overall geometry of the unit is a distorted tetrahedron as determined by the arrangement that the aldehyde functionalities make with respect to each other. This unit when reticulated with linear linkers was expected to form a square net (Figure $\mathrm{bb}$ ). We also recognized that the distorted tetrahedral units, SFTB, should minimize $\pi-\pi$ interactions and
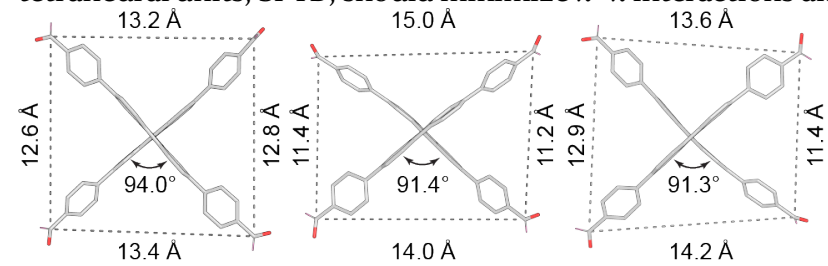

Figure 2. Single crystal structure of SFTB showed three independent as-symmetric units with different edge lengths and dihedral angles. 
a)

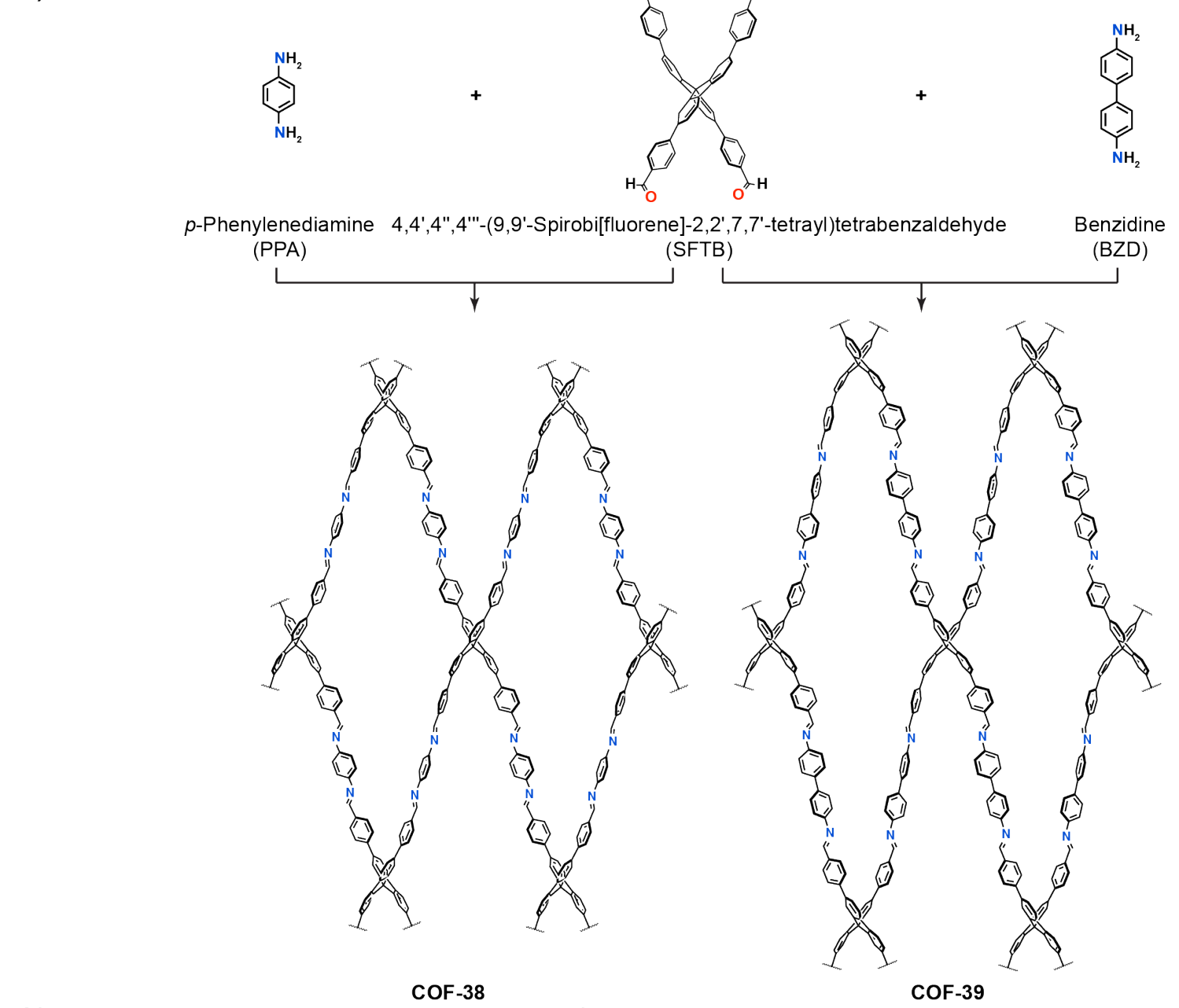

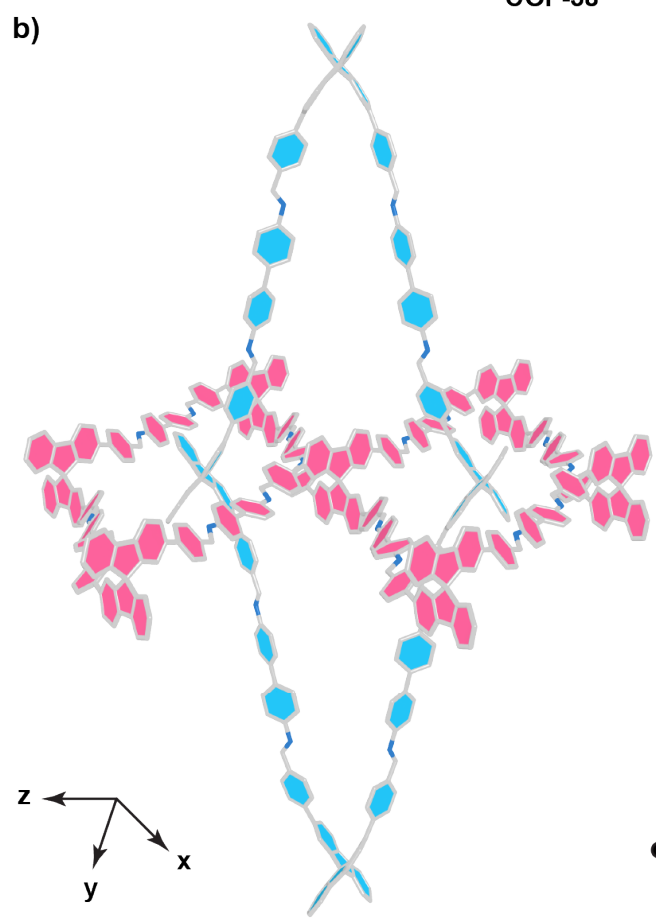

Entanglement of square nets

c)
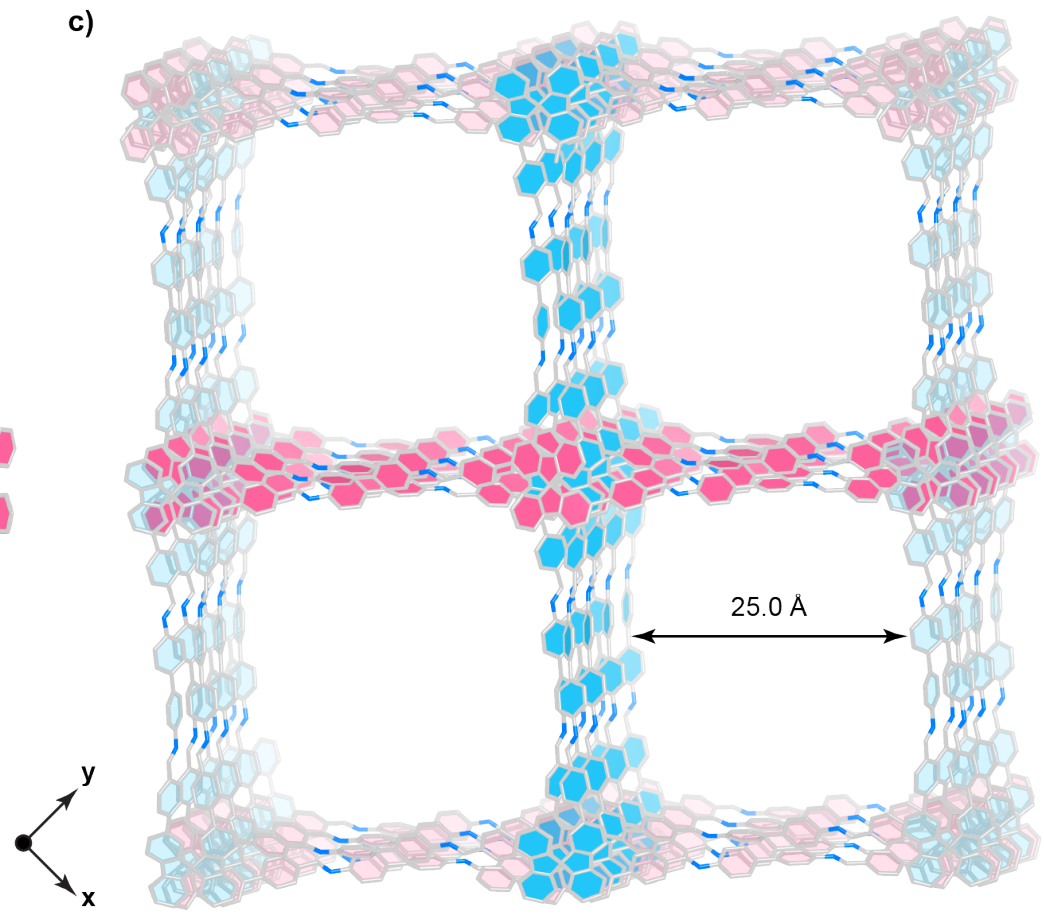

COF-39

Figure 3. Strategy of constructing $3 \mathrm{D}$ COFs through entanglement of squares. (a) 4,4',4",4"'-(9,9'-Spirobi[fluorene]-2,2',7,7'tetrayl)-tetrabenzaldehhyde (SFTB) reacted with $p$-phenylenediamie (PPA) and benzidine (BZD) to form COF-38 and COF-39, respectively. (b) Entanglement of squares in COF-39. (c) The overall 3D structure showed $1 \mathrm{D}$ square channels in COF-39. 

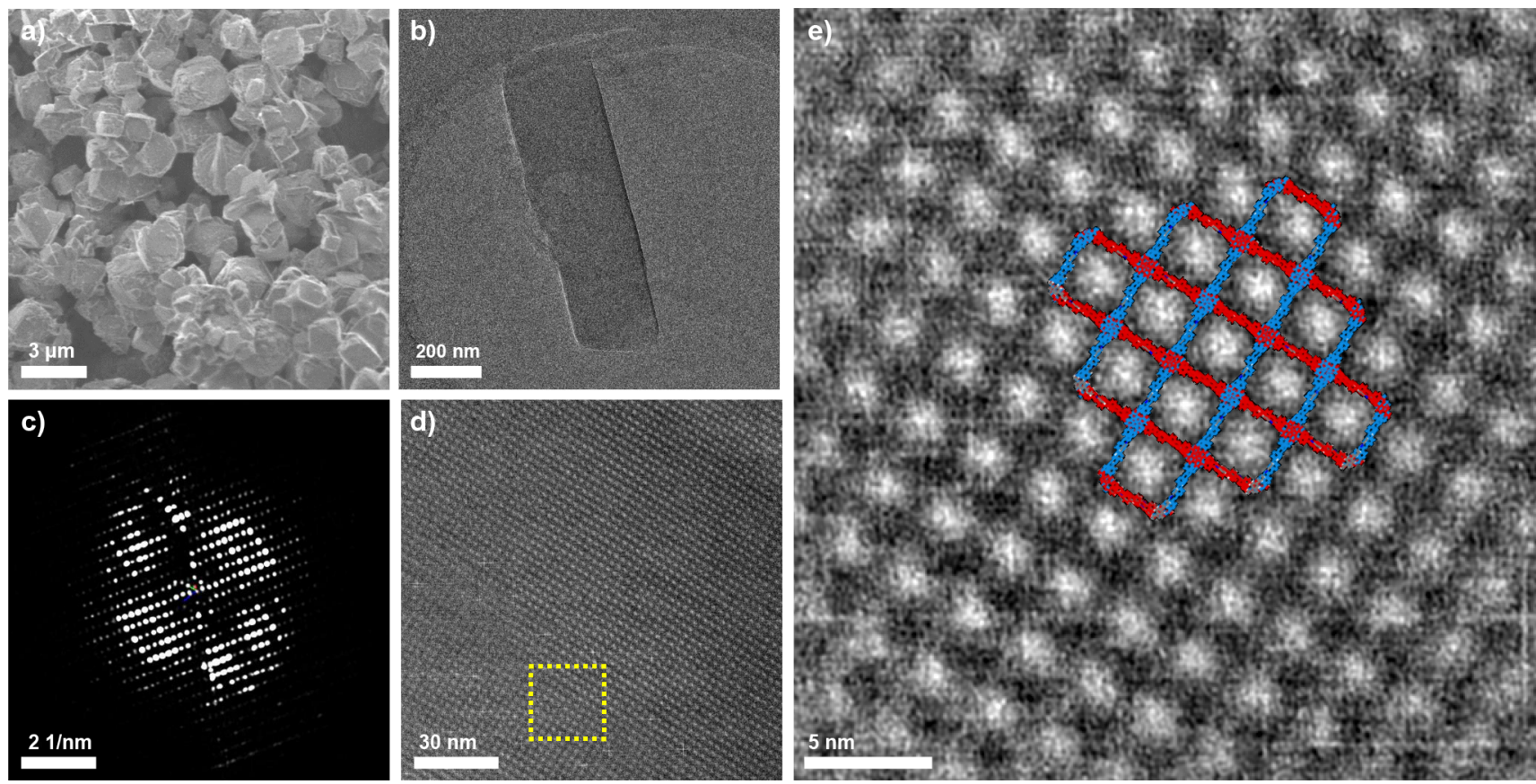

Figure 4. Electron microscopy data of COF-39. (a) Scanning electron microscopy (SEM) image of COF-39 presented the conglomerate of prism-shaped crystals. (b) Corresponding single crystal used for $3 \mathrm{D}$ ED data collection. (c) $2 \mathrm{D}$ projections of the $3 \mathrm{D}$ reciprocal lattice of COF-39 reconstructed from 3D ED data. (d) Average background subtraction filter (ABSF) high-resolution TEM (HRTEM) image of COF-39 taken along [oo1] direction. (e) Magnified view of the highlighted area of (d) overlaid with a structure model showed good agreement along [oo1].

therefore encourage the formation of square entanglements rather than the usual stacking of layers. We have successfully implemented this strategy by condensation of SFTB and $p$ phenylenediamine (PPA) or benzidine (BZD) to make ${ }_{3} \mathrm{D}$ COFs (COF-38, [(SFTB)(PPA $\left.)_{2}\right]_{\text {imine }}$ and COF-39, $\left[(\mathrm{SFTB})(\mathrm{BZD})_{2}\right]_{\text {imine, }}$ respectively) composed of entanglement of $2 \mathrm{D}$ nets (Figure $1 \mathrm{C}$ ). We believe that our strategy widens the scope of possible COF structures where COF-38 and COF-39 are the first examples of entangled $2 \mathrm{D}$ COFs of any topology. It is worth mentioning that exhaustive efforts were undertaken to grow large crystals for $\mathrm{COF}-38$ but only submicrocrystals were obtained. However, we successfully synthesized highly crystalline COF-39 sample for electron microspopy analysis and elucidated its crystal structure. Therefore, we will discuss the synthesis and characterizations of COF-39 in the following content while the synthesis and characterizations of isoreticular $\mathrm{COF}-38$ are detailed in the supporting information (SI).

We synthesized SFTB using a slightly modified literature procedure and successfully crystallized this linker (SI, Section S2). We also determined the single crystal X-ray structure of SFTB and found that it has a distorted tetrahedral geometry (SI, Section S3). Single crystal structure of SFTB (Figure 2) clearly showed three independent as-symmetric units with different edge lengths measured counterclockwise from the aldehyde carbon to the adjacent one. The edge lengths were in the range between 11.2 $\AA$ and 15.0 A. The average dihedral angle of two spirobi[fluorene] rings was $92.2^{\circ}$. The ratio of the rectangle sides in SFTB linker was around 1.2, indicating its distorted tetrahedral geometry. The rectangular rather than square SFTB shape played a role in producing the staggered COF framework and enhancing the flexibility of the extended structure (Figure $1 \mathrm{~b}$ ). SFTB was then reacted with linear amine-functionalized linkers PPA and BZD to form two $2 \mathrm{D}$ entangled COFs, namely COF-38 and COF-39, respectively (Figure $3 a)$.

COF-39 was synthesized solvothermally by reacting the linkers SFTB and BZD in a 1:2 molar ratio in trichlorobenzene with aqueous trifluoroacetic acid as a catalyst (SI, Section S2). The reaction mixture was sealed in a Pyrex tube and heated to $120{ }^{\circ} \mathrm{C}$ for $3 \mathrm{~d}$. COF-39, a yellow precipitate formed at the bottom of the tube, was isolated by centrifugation and washed by Soxhlet extraction with anhydrous tetrahydrofuran for $18 \mathrm{~h}$ to remove unreacted reactants. The material was then solvent exchanged with supercritical carbon dioxide and was finally activated under dynamic vacuum at room temperature for $1 \mathrm{~h}$ and then at $150{ }^{\circ} \mathrm{C}$ for $5 \mathrm{~h}$. COF-39 was fully characterized by powder X-ray diffraction (PXRD), Fourier-transform infrared (FT-IR) spectroscopy, solid- and solution-state nuclear magnetic resonance (NMR) spectroscopies, elemental analysis (EA), thermogravimetric analysis (TGA), nitrogen sorption, scanning electron microscopy (SEM), and transmission electron microscopy (TEM) (SI, Sections S2-S9).

The FT-IR spectroscopy indicated the formation of imine linkages of COF-39 at $v_{\mathrm{C}=\mathrm{N}}=1625 \mathrm{~cm}^{-1}$ and the consumption of aldehyde starting material since there was no identifiable $v_{\mathrm{C}=\mathrm{O}}$ aldehyde stretching vibration at $1695 \mathrm{~cm}^{-1}$ remaining (SI, Section $\mathrm{S}_{4}$ ). Further confirmation of imine-linkage formation in COF-39 was shown by ${ }^{13} \mathrm{C}$ cross-polarization magic angle spinning (CP-MAS) NMR spectroscopy (SI, Section $\mathrm{S}_{5}$ ) where the characteristic $\mathrm{C}=\mathrm{N}$ imine resonances at $160 \mathrm{ppm}$ was observed. Additionally, the disappearance of resonances above $190 \mathrm{ppm}$ indicated that SFTB was fully converted into COF-39 by the imine condensation reaction. Analysis by solution-state NMR of acidic-digested COF-39 showing a stoichiometric ratio of 1:2 of SFTB:BZD, indicating there were minimal defects in COF- 
39 structure (SI, Section $\mathrm{S}_{5}$ ). CHN analysis of COF-39 corresponded to a reticular formula of $\left[(\mathrm{SFTB})(\mathrm{BZD})_{2}\right]_{\text {imine }}$ (Calcd for $\mathrm{C}_{77} \mathrm{H}_{48} \mathrm{~N}_{4} \cdot 3 \mathrm{H}_{2} \mathrm{O}$ : C, 85.37; H, 5.02; N, 5.17\%. Found: C, 85.94; $\mathrm{H}, 5.23$; N, 5.29\%; SI, section $\mathrm{S}_{2}$ ) indicating that all water molecules were not fully removed in COF-39 (ca. 5.0\%). This data agreed with the FT-IR spectroscopy and TGA profile. The thermal stability of COF-39 was studied by TGA measured under $\mathrm{N}_{2}$ atmosphere, and the onset in thermal decomposition of COF-39 was found to be around $480^{\circ} \mathrm{C}$ (SI, section S6).

The SEM micrographs of COF-39 showed a homogeneous morphology of $1-\mu \mathrm{m}$ prism-shaped crystals (Figure $4 \mathrm{a}$ ) formed by conglomerate of thin and long needle-like crystals (SI, section $\mathrm{S}_{7}$ ). The homogeneous morphology of COF-39 indicates phase purity, as also shown by TEM analysis (Figure $4 \mathrm{~b}-\mathrm{e}$, and SI, S8). Due to its high crystallinity, COF-39 exhibited the PXRD pattern with sharp peaks and low background (Figure 5). We were able to index at least 30 peaks from the PXRD pattern of COF-39 (SI, section S8). The indexing result suggested that COF-39 crystallized in Tetragonal Bravais lattice with space group of $I_{4}$ or $I_{4}$ which was in line with our hypothesis of having a $3 \mathrm{D}$ rather than a $2 \mathrm{D}$ COF structure.

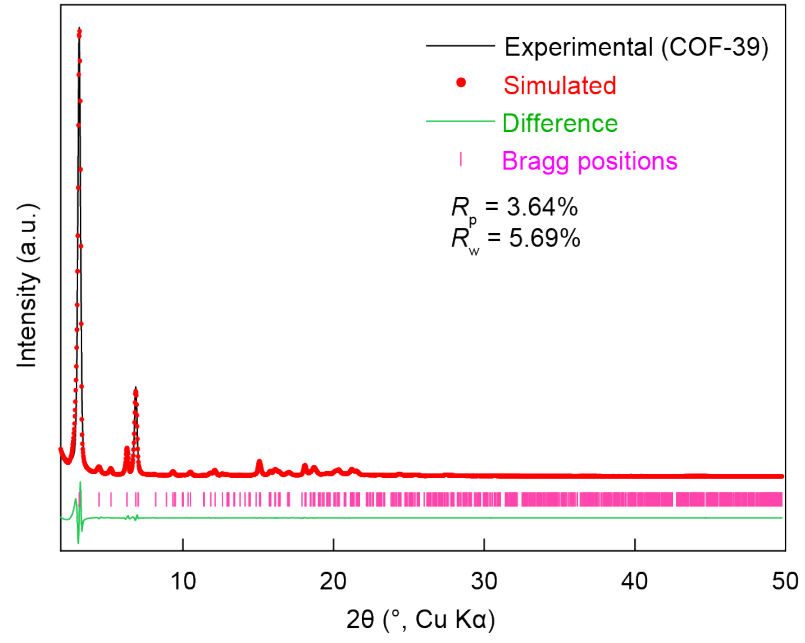

Figure 5. Pawley fitting (red circles) of experimental PXRD patterns (black lines) of activated sample of COF-39.

For TEM analysis (SI, Section S8), COF-39 was highly dispersed into ethanol and several droplets of the suspension were subsequently dropped onto a carbon-coated copper grid for volatilization under ambient conditions. $3 \mathrm{D}$ electron diffraction (3D ED) data of COF-39 were collected to further elucidate the crystal structure. ${ }^{28} \mathrm{~A}$ typical $3 \mathrm{D}$ ED dataset with a resolution about $1.9 \AA$ is shown in projections (Figure $4 \mathrm{c}$ ). From the reconstructed $3 \mathrm{D}$ reciprocal lattice, a body-centered Tetragonal Bravais lattice was identified (SI, Section S8) and the unit cell parameters were determined as $a=39 \cdot 9 \AA$, $c=19 \cdot 3$ $\AA$, and $V=30654.6 \AA^{3}$.

Based on the information obtained from ${ }_{3} \mathrm{D}$ ED, we tried to build the structural model of COF-39 in Materials Studio ${ }^{\circledR}$ $8.0^{29}$ using the Tetragonal space group $I_{4}, I_{-} 4$, and $I_{4} / \mathrm{m}$. However, only the structural model built using $I_{4}$ as the space group showed chemically reasonable structure and the simulated PXRD pattern showed great alignment to the experimental one (SI, Section S8). The unit cell parameters were then refined by Pawley fitting against the experimental PXRD pattern: $a=39.8 \AA, c=19.2 \AA, V=30413 \AA^{3}$ with very low reliability factors $\left(R_{\mathrm{p}}=3.64 \%, R_{\mathrm{wp}}=5.69 \%\right.$; Figure 5$)$. More importantly, the calculated PXRD pattern of the built structure matched well with the experimental data of COF-39 (SI, Section S8).

According to the structural model, 2D sql layers (Figure za) were formed by stitching together SFTB and BZD, followed by the staggered stacking (AB-stacking form) of these formed layers along the $c$ axis. The edge lengths of SFTB in COF-39 were found to be $8.4 \AA \times 16.4 \AA$. The rectangle sides ratio was 2.o, larger than that of SFTB alone, indicating the high flexibility of the resulting framework. The dihedral angle of two spirobi[fluorene] rings in COF-39 was 92.5 (SI, Section S8). The wide opening of the rhombic windows within each of the layers and the high flexibility of building units triggered the entanglement of another identical net (Figure 3b). Accordingly, one set of the staggered stacked layers entangled perpendicularly with another identical set to give rise to the final $3 \mathrm{D}$ framework adopting $(4,4)$-c entanglement with $1 \mathrm{D}$ square channel along [oo1] direction (Figure 3c). The crystal structure of COF-39 is completely different from that claimed to be a $3 \mathrm{D}$ COF with 7-fold dia topology reported by Liu and co-workers, ${ }^{30}$ even though similar building units were used.

High-resolution TEM images (HRTEM) were also taken to further gain insights into the crystal structure of COF-39. Indeed, the overlay of the structural model with the experimentally obtained HRTEM images along [0o1] and [111] both showed good agreement (Figure 4e, and SI, Section S8).

The permanent porosity of COF-39 was studied using $\mathrm{N}_{2}$ sorption analysis at $77 \mathrm{~K}$. Similar to entangled $3 \mathrm{D}$ COFs, ${ }^{16,19}$ COF-39 exhibited a Type I isotherm with a Brunauer-Emmett-Teller (BET) surface area of $813 \mathrm{~m}^{2} \mathrm{~g}^{-1}$ (SI, Section S9).

\section{ASSOCIATED CONTENT}

The supporting information is available free of charge via the Internet at http://pubs.acs.org.

Synthesis and characterization details of COF-38 and COF-39 including elemental analysis, Fourier-transform infrared spectroscopy, nuclear magnetic resonance spectra, powder X-ray diffraction analysis data, electron microscopy data, computational modeling, $\mathrm{N}_{2}$ sorption measurements, and thermogravimetric analysis (PDF).

\section{AUTHOR INFORMATION}

\section{Corresponding Author}

*O.M.Y.: yaghi@berkeley.edu

\section{Author Contributions}

F.J. and O.M.Y. conceived the idea. F.J. synthesized the COFs. F.J. and H.L.N. interpreted the data and discussed the structures of COFs. X.H., H.L.N., and F.J. modeled the structures. Z.Z and Y.M. measured SEM, HRTEM and 3 D ED. X.P. measured the SXRD of SFTB. C.Z. collected WAXS data of COF-38. O.M.Y. directed the project. The manuscript was written through contributions of all authors. ${ }^{\ominus}$ F.J. and H.L.N. contributed equally.

\section{Funding Sources}

King Abdulaziz City for Science and Technology (Center of Excellence for Nanomaterials and Clean Energy Applications); DOD Advanced Research Projects Agency Award Number HRoo112020038; DOE Office of Science User Facility (contract no. DE-ACo2-05CH11231); National Natural Science 
Foundation of China (No. 21875140) and CћEM SPST, ShanghaiTech University (\#EMo2161943)

\section{Notes}

The authors declare no competing financial interests.

\section{ACKNOWLEDGMENT}

We thank Dr. Cornelius Gropp (Yaghi Research Group) and Prof. George Lisensky (Beloit College) for valuable discussion. We thank Dr. Tianqiong Ma for the SEM measurement of COF-38. We acknowledge King Abdulaziz City for Science and Technology as part of a joint KACST-UC Berkeley collaboration and UAE University as part of a joint UAEU-UC Berkeley collaboration. We thank the financial support from DOD Advanced Research Projects Agency Award Number HRool12020038. C.Z thanks the financial support for the PXRD measurements at beamline 7.3.3 of the Advanced Light Source from DOE Office of Science User Facility under contract no. DE-AC02-05CH11231. Z.Z. and Y.M acknowledges the financial support from National Natural Science Foundation of China (No. 21875140) and CћEM SPST, ShanghaiTech University (\#EMo2161943) for TEM measurements. We acknowledge the NIH (Grant S10-RRo27172) for financial support of the X-ray crystallographic facility at UC Berkeley, and Dr. Nicholas Settineri for the support on using the facility. We acknowledge the College of Chemistry Nuclear Magnetic Resonance Facility for resource instruments, which are partially supported by NIH S1oODo24998, and staff assistance from Dr. Hasan Celik and Dr. Alicia Lund.

\section{REFERENCES}

(1) Yaghi, O. M.; Kalmutzki, M. J.; Diercks, C. S. Introduction to Reticular Chemistry: Metal-organic frameworks and covalent organic frameworks; Wiley-VCH: Weinheim, 2019.

(2) Diercks, C. S.; Yaghi, O. M. The atom, the molecule, and the covalent organic framework. Science 2017, 355, 923.

(3) Feng, X.; Ding, X.; Jiang, D. Covalent Organic Frameworks. Chem. Soc. Rev. 2012, 41, 6010-6022.

(4) Kandambeth, S.; Dey, K.; Banerjee, R. Covalent Organic Frameworks: Chemistry beyond the Structure. J. Am. Chem. Soc. 2019, 141, 1807-1822.

(5) Lohse, M. S.; Bein, T. Covalent Organic Frameworks: Structures, Synthesis, and Applications. Adv. Funct. Mater. 2018, 28, 1705553

(6) Hasse, F.; Lotsch, B. V. Solving the COF trilemma: towards crystalline, stable and functional covalent organic frameworks. Chem. Soc. Rev. 2020, 49, 8469-8500.

(7) Cote, A. P.; Benin, A. I.; Ockwig, N. W.; O’Keeffe, M.; Matzger, A. J.; Yaghi, O. M. Porous, Crystalline, Covalent Organic Frameworks. Science 2oo5, 310, 1166-1170.

(8) Waller, P. J.; Gandara, F.; Yaghi, O. M. Acc. Chem. Res. 2015, 48, 3053-3063.

(9) Geng, K.; He, T.; Liu, R.; Dalapati, S.; Tian, T.; Li, Z.; Tao, S.; Gong, Y.; Jiang, Q.; Jiang, D. Covalent Organic Frameworks: Design, Synthesis, and Functions. Chem. Rev. 2020, 120, 8814-8933.
(10) Guan, X.; Chen, F.; Fang, Q.; Qiu, S. Design and applications of three dimensional covalent organic frameworks. Chem. Soc. Rev. 2020, 49, 1357-1384.

(11) Ma, X.; Scott, T. F. Approaches and Challenges in the Synthesis of Three-Dimensional Covalent-Organic Frameworks. Commun. Chem. 2018, 1, 98.

(12) Nguyen, H. L. Reticular design and crystal structure determination of covalent organic frameworks. Chem. Sci. 2021, 12, 8632-8647.

(13) El-kaderi, H. M.; Hunt, J. R.; Mendoza-cortes, J. L.; Cote, A. P.; Taylor, R. E.; O'Keeffe, M.; Yaghi, O. M. Designed Synthesis of $3 \mathrm{D}$ Covalent Organic Frameworks. Science 2o17, 316, 268-272.

(14) Liu, Y.; Ma, Y.; Zhao, Y.; Sun, X.; Gandara, F.; Furukawa, H.; Liu, Z.; Zhu, H.; Zhu, C.; Suenaga, K.; Oleynikov, P.; Alshammari, A. S.; Zhang, X.; Terasaki, O.; Yaghi, O. M. Weaving of Organic Threads into a Crystalline Covalent Organic Framework. Science 2016, 351, 365-369.

(15) Lin, G.; Ding, H.; Yuan, D.; Wang, B.; Wang, C. A PyreneBased, Fluorescent Three-Dimensional Covalent Organic Framework. J. Am. Chem. Soc. 2016, 138, 10, 3302-3305.

(16) Ma, T.; Kapustin, E. A.; Yin, S. X.; Liang, L.; Zhou, Z.; Niu, J.; Li, L.; Wang, Y.; Su, J.; Li, J.; Wang, X.; Wang, W.; Wang, W.; Sun, J.; Yaghi, O. M. Single-crystal x-ray diffraction structures of covalent organic frameworks. Science 2018, 361, 48-52.

(17) Zhu, Q.; Wang, X.; Clowes, R.; Cui, P.; Chen, L.; Little, M. A.; Cooper, A. I. 3D Cage COFs: A Dynamic Three dimensional covalent organic framework with high-connectivity organic cage nodes. J. Am. Chem. Soc. 2020, 142, 16842-16848.

(18) Gropp, C.; Ma, T.; Hanikel, N.; Yaghi, O. M. Design of higher valency in covalent organic frameworks. Science 2020, 370, eabd6406.

(19) Li, H.; Ding, J.; Guan, X.; Chen, F.; Li, C.; Zhu, L.; Xue, M.; Yuan, D.; Valtchev, V.; Yan, Y.; Qiu, S.; Fang, Q. Three-Dimensional Large-Pore Covalent Organic Framework with stp Topology. J. Am. Chem. Soc. 2020, 142, 31, 13334-13338.

(20) Xie, Y.; Li, J.; Lin, C.; Gui, B.; Ji, C.; Yuan, D.; Sun, J.; Wang, C. Tuning the Topology of Three-Dimensional Covalent Organic Frameworks via Steric Control: From pts to Unprecedented ljh. J. Am. Chem. Soc. 2021, 143, 7279-7284.

(21) Li. Z.; Sheng, L.; Wang, H.; Wang, X.; Li, M.; Xu, Y.; Cui, H.; Zhang, H.; Liang, H.; Xu, H.; He, X. Three-Dimensional Covalent Organic Framework with ceq Topology. J. Am. Chem. Soc. 2021, $143,1,92-96$.

(22) Uribe-Romo, F. J.; Hunt, J. R.; Furukawa, H.; Klock, C.; O'Keeffe, M.; Yaghi, O. M. A Crystalline Imine-Linked 3-D Porous Covalent Organic Framework.J. Am. Chem. Soc. 2009, 131, 13, $4570-4571$.

(23) Ma, T.; Kapustin, E. A.; Yin, S. X.; Liang, L.; Zhou, Z.; Niu, J.; Li, L.; Wang, Y.; Su, J.; Li, J.; Wang, X.; Wang, W.; Wang, W.; Sun, J.; Yaghi, O. M. Single-crystal x-ray diffraction structures of covalent organic frameworks. Science, 2018, 52, 48-52. 
(24) Ma, T.; Li, J.; Niu, J.; Zhang, L.; Etman, A. S.; Lin, C.; Shi, D.; Chen, P.; Li, L.; Du, X.; Sun, J.; Wang, W. J. Am. Chem. Soc. 2018, 140, 22, 6763-6766.

(25) Carlucci, L.; Ciani, G.; Proserpio, D. M.; Mitina, T. G.; Blatov, V. A. Entangled Two-Dimensional Coordination Networks: A General Survey. Chem. Rev. 2014, 114, 7557-7580.

(26) Haase, F.; Gottschling, K.; Stegbauer, L.; Germann, L. S.; Gutzler, R.; Duppel, V.; Vyas, V. S.; Kern, K.; Dinnebier, R. E.; Lotsch, B. V. Tuning the stacking behaviour of a $2 \mathrm{D}$ covalent organic framework through non-covalent interactions. Mater. Chem. Front. 2017, 1, 1354-1361.
(27) Batten, S. R.; Robson, R. Interpenetrating Nets: Ordered, Periodic Entanglement. Angew. Chem. Int. Ed. 1998, 37, 1460-1494.

(28) Gemmi, M.; Oleynikov, P. Scanning Reciprocal Space for Solving Unknown Structures: Energy Filtered Diffraction Tomography and Rotation Diffraction Tomog-raphy Methods. Z. Kristallogr. Cryst. Mater. 2013, 228, 51-58.

(29) Dassault Systemes BIOVIA, Materials Studio 8.o, Dassault Systemes, San Diego, 2014.

(30) Liu, Y.; Li, X.; Wang, S.; Cheng, T.; Yang, H.; Liu, C.; Gong, Y.; Lai, W.; Huang, W. Self-templated synthesis of uniform hollow spheres based on highly conjugated three-dimensional covalent organic frameworks. Nat. Commun. 2020, 11, 5561. 
Table of Content
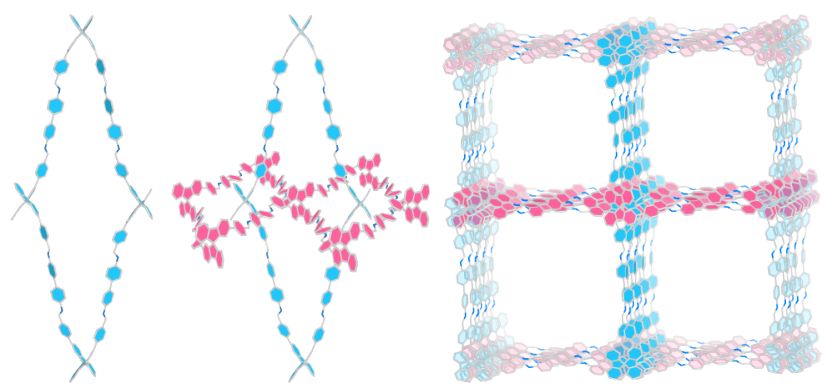

Square net Entanglement of square nets

COF-39 